\title{
Isolation and Identification of Triterpenoids and Sterols from the Flowers of Chionanthus retusus Lindl. \& Paxton
}

\author{
Jae-Woo Jung $\cdot$ Kyeong-Hwa Seo $\cdot$ Eun-Ji Oh $\cdot$ Dae-Young Lee " Nam-In Baek* (D)
}

\section{이팝나무(Chionanthus retusus Lindl. \& Paxton) 꽃으로부터 Triterpenoid 및 Sterol 화합물의 분리 및 동정}

정재우 ·서경화 · 오은지 · 이대영 · 백남인*

Received: 28 April 2015 / Accepted: 2 June 2015 / Published Online: 30 September 2015

(C) The Korean Society for Applied Biological Chemistry 2015

\begin{abstract}
The flowers of Chionanthus retusus Lindl. \& Paxton were extracted with $80 \%$ aqueous $\mathrm{MeOH}$ at room temperature. The concentrated extract was partitioned as EtOAc, $n-\mathrm{BuOH}$, and $\mathrm{H}_{2} \mathrm{O}$ fractions. From the EtOAc fraction, two triterpenoids and two sterols were isolated using the repeated silica gel $\left(\mathrm{SiO}_{2}\right)$ and octadecyl $\mathrm{SiO}_{2}$ (ODS) column chromatographies. According to the results of physico-chemical and spectroscopic data including nuclear magnetic resonance, mass spectrometry, and infrared. The chemical structures of the compounds were respectively determined as ursolic acid (1), corosolic acid (2), $\beta$-sitosterol (3), and daucosterol (4). All the compounds were isolated for the first time from the flowers of Chionanthus retusus Lindl. \& Paxton.
\end{abstract}

Keywords Chionanthus retusus Lindl. \& Paxton - flower · nuclear magnetic resonance $\cdot$ sterol $\cdot$ triterpenoid

J.-W. Jung $\cdot$ K.-H. Seo $\cdot$ E.-J. Oh $\cdot$ N.-I. Baek

Graduate School of Biotechnology \& Department of Oriental Medicine Biotechnology, Kyung Hee University, Yongin 446-701, Republic of Korea

D.-Y. Lee

Department of Herbal Crop Research, National Institute of Horticultural and Herbal Science, RDA, Eumseong 369-873, Republic of Korea

*Corresponding author (N.-I. Baek: nibaek@khu.ac.kr)

This is an Open Access article distributed under the terms of the Creative Commons Attribution Non-Commercial License (http://creativecommons. org/licenses/by-nc/3.0/) which permits unrestricted non-commercial use, distribution, and reproduction in any medium, provided the original work is properly cited.
서 론

이팝나무(Chionanthus retusus Lindl. \& Paxton)는 물푸레나무 과(Oleaceae)의 낙엽활엽교목으로서 우리나라 전역에 분포하고 있으며, 타이완, 중국, 일본에도 자생하고 있다. 높이는 약 $20 \mathrm{~m}$ 까지 자라고, 꽃은 자웅이주(雌雄異株)이며 새가지 끝에 원추화 서(圓錐花序)가 길이 $5-10 \mathrm{~cm}$ 로 5 월에 백색으로 핀다. 열매는 핵과(核果)로 타원형이며 길이는 1-1.5 cm로 9-10월에 벽흑색 으로 익는다(Lee 등, 1996). 이팝나무는 이전 조선시대에 쌀밥 의 의미인 '이밥'으로 나무의 가지 끝의 탐스런 꽃송이가 사발 에 소복이 담긴 흰 쌀밥처럼 보여 ‘이밥나무'라고 불렸으며, 이 후 시간이 지나 ‘이팝’으로 변했다고 한다. 예로부터 민간요법 으로 이팝나무 잎을 설사, 중풍, 해열의 치료로 사용했다고 한 다(Kim, 1996).

이팝나무로부터 지금까지 분리 보고된 성분으로는 잎으로부터는 phenolic 화합물인 (7R,8R)-guaiacylglycerol과 2-(4-hydroxyphenyl) ethanol과 oleuropein의 secoiridoid glycoside 화합물과, scopoletin 의 coumarin 화합물이 있으며, phillyrin 등의 lignan 화합물과 kaempferol 등의 flavonoid 화합물이 있다(Kwak 등, 2009). 또 한 분리된 화합물에 대하여 Human tumor cell lines (A549, SK-OV-3, SK-MEL-2, HCT15)의 세포독성 활성연구가 보고되 어 있다(Kwak 등, 2009).

이팝나무는 관상수로서 우리나라에서 널리 자생하여 원료의 확보가 용이하고, 특히 꽃으로부터는 성분과 활성에 대한 연구 는 미비하다. 따라서 저자는 이팝나무 꽃으로부터 이차대사산물 을 분리하고 각 물질에 대한 성분분석과 여러가지 생리활성에 관련한 연구를 진행하여 이팝나무 꽃의 의약품 및 화장품 소재 로서의 활용 방안을 모색하고자 실험을 수행하였다. 이팝나무 꽃을 methanol $(\mathrm{MeOH})$ 로 추출하고 용매 분획한 후 얻어진 ethyl acetate (EtOAc) 및 $n$-butyl alcohol $(n-\mathrm{BuOH})$ 분획에 대하여 TLC 
를 확인한 결과 terpenoid, steroid, flavonoid 및 flavonoid glycoside로 예상되는 화합물이 존재함을 확인할 수 있었다. 기 존에 보고되어 있지 않은 terpenoid와 steroid를 분리하고자 비극 성 분획인 EtOAc 분획에 대하여 silica gel $\left(\mathrm{SiO}_{2}\right)$ 및 octadecyl $\mathrm{SiO}_{2}$ (ODS) column chromatography를 반복 수행하여 2종의 triterpenoid 화합물과 2종의 sterol 화합물을 분리 하였으며, nuclear magnetic resonance (NMR), infrared (IR), mass spectrometry (MS) 등의 스펙트럼 데이터를 해석하여 화학 구조를 동정하였 다. 분리한 4종의 화합물은 이팝나무 꽃으로부터 이번 실험에 서 처음 분리 되었다.

\section{재료 및 방법}

실험재료. 본 실험에 사용한 이팝나무(Chionanthus retusus Lindl. \& Paxton) 꽃은 2014년 8월 경희대학교 국제캠퍼스 산 지에서 채집하였고, 우석대학교 약학대학 김대근 교수가 동정하 였으며, 표본시료(KHU-NPCL-201408)는 경희대학교 천연물화 학실에 보관되어 있다.

시약 및 기기. Open column chromatography (c.c.)용 $\mathrm{SiO}_{2}$ 은 Kiesel gel 60 (Merck, Germany), 그리고 ODS는 LiChroprep RP-18 (Merck)을 사용하였다. Thin layer chromatography (TLC)는 Kieselgel $60 \quad \mathrm{~F}_{254}$ 와 RP-18 $\mathrm{F}_{254} \mathrm{~S}$ (Merck)를 사용하였 고, TLC 상의 물질 검출에는 UV lamp는 Spectroline (Model ENF-240 C/F, Spectronics Corporation, USA)로 확인 후, $10 \%$ $\mathrm{H}_{2} \mathrm{SO}_{4}$ 를 분무한 후 가열하여 발색양상을 조사하였다. NMR spectrum은 Varian Inova AS 400 (Varian, USA)으로 측정하였 고, IR spectrum은 Perkin model 599B (Waltham, USA)로 측정하였다. Electronic ionization mass spectrometer (EI/MS) spectrum은 JEOL JMS 505-WA (JEOL, Japan)로 측정하였다. 융점은 Fisher-Johns 융점 측정기(Fisher Scientific, USA)를 사 용하여 측정하였고, 미보정하였다.

추출 및 분흭. 건조된 이팝나무 꽃 $315 \mathrm{~g}$ 을 $80 \% \mathrm{MeOH}$ 수용 액 $(23 \mathrm{~L} \times 3)$ 으로 실온에서 24 시간 3 회 추출한 후 여과지로 여과 하였다. 얻어진 추출물을 감압 농축하여 $\mathrm{MeOH}$ 추출물 $(145 \mathrm{~g})$ 을 얻었다. $\mathrm{MeOH}$ 추출물은 $\mathrm{H}_{2} \mathrm{O}(2 \mathrm{~L})$ 와 EtOAc $(2 \mathrm{~L} \times 3)$ 로 분 배 추출하였고, 다시 $\mathrm{H}_{2} \mathrm{O}$ 층을 $n-\mathrm{BuOH}(1.8 \mathrm{~L} \times 2)$ 로 분배 추출 하였다. 각 층을 감압농축하여 $\mathrm{EtOAc}$ 분획 $(\mathrm{CFE}, 27 \mathrm{~g}), n$ $\mathrm{BuOH}$ 분획 $(\mathrm{CFB}, 24 \mathrm{~g})$ 및 $\mathrm{H}_{2} \mathrm{O}$ 분획 $(\mathrm{CFW}, 94 \mathrm{~g})$ 을 얻었다.

EtOAc 분획으로부터 triterpenoid 및 sterol의 분리. EtOAc 분 획 $(27 \mathrm{~g})$ 으로부터 $\mathrm{SiO}_{2}$ c.c. $\left(11 \times 12 \mathrm{~cm}\right.$, chloroform $\left(\mathrm{CHCl}_{3}\right)-\mathrm{MeOH}=$ $40: 1 \rightarrow 10: 1 \rightarrow 5: 1 \rightarrow 2: 1 \rightarrow 1: 1$, 각 $2.6 \mathrm{~L}$ )를 실시하여 16 개의 분획 물(CFE-1-CFE-16)을 얻었다. MRE-3 [3 g, elution volume/total volume $(\mathrm{Ve} / \mathrm{Vt})=0.131-0.172]$ 을 $\mathrm{SiO}_{2}$ c.c. $(3.5 \times 14 \mathrm{~cm}, n$-hexane$\mathrm{EtOAc}=4: 1$, 각 $1.2 \mathrm{~L}$ )를 실시하여 11 개의 분획(CFE-3-1-CFE-3$11)$ 으로 나누었고, 그 중 화합물 3 (CFE-3-5, $80 \mathrm{mg}, \mathrm{Ve} / \mathrm{Vt}=$ 0.012-0.015, TLC $\left(\mathrm{SiO}_{2} \mathrm{~F}_{254}\right) \mathrm{R}_{\mathrm{f}}$ 0.50, $n$-hexane-EtOAc=3:1)과 화합물 1 (CFE-3-8, $821 \mathrm{mg}, \mathrm{Ve} / \mathrm{Vt}=0.257-0.678$, TLC (ODS $\left.\mathrm{F}_{254} \mathrm{~S}\right)$ $\mathrm{R}_{\mathrm{f}} 0.69$, acetone- $\left.\mathrm{H}_{2} \mathrm{O}=10: 1\right)$ 을 분리하였다. CFE-5 (3.2 g, $\mathrm{Ve} / \mathrm{Vt}=$ $0.360-0.415)$ 을 ODS c.c. $\left(5.5 \times 7 \mathrm{~cm}, \mathrm{MeOH}-\mathrm{H}_{2} \mathrm{O}=4: 1,1.7 \mathrm{~L}\right)$ 를 실시하여 13개의 분획(CFE-5-1-CFE-5-13)으로 나누었고, 그 중 화합물 2 (CFE-5-10, $800 \mathrm{mg}, \mathrm{Ve} / \mathrm{Vt}=0.476-0.882$, TLC (ODS $\mathrm{F}_{254} \mathrm{~S}$ ) $\mathrm{R}_{\mathrm{f}}$ 0.58, acetone- $\left.\mathrm{H}_{2} \mathrm{O}=10: 1\right)$ 을 분리하였다. CFE-8 (400 mg, $\mathrm{Ve} / \mathrm{Vt}=0.489-0.572)$ 을 ODS c.c. $\left(4 \times 7 \mathrm{~cm}\right.$, acetone- $\left.\mathrm{H}_{2} \mathrm{O}=2: 1,2 \mathrm{~L}\right)$
Table $1{ }^{13} \mathrm{C}-\mathrm{NMR}$ data $(100 \mathrm{MHz})$ of compounds (1-4) from the flowers of Chionanthus retusus Lindl. \& Paxton

\begin{tabular}{|c|c|c|c|c|}
\hline No. of Carbon & Compound 1 & Compound $\mathbf{2}$ & Compound $\mathbf{3}$ & Compound $\mathbf{4}$ \\
\hline 1 & 38.62 & 47.93 & 37.31 & 37.33 \\
\hline 2 & 28.24 & 68.58 & 31.64 & 29.81 \\
\hline 3 & 78.15 & 83.79 & 71.69 & 78.18 \\
\hline 4 & 39.02 & 39.78 & 42.24 & 39.88 \\
\hline 5 & 55.84 & 55.91 & 140.55 & 140.85 \\
\hline 6 & 18.38 & 18.82 & 121.69 & 121.92 \\
\hline 7 & 32.98 & 33.51 & 31.88 & 31.78 \\
\hline 8 & 41.64 & 40.04 & 31.88 & 31.62 \\
\hline 9 & 47.12 & 48.09 & 50.08 & 50.48 \\
\hline 10 & 36.97 & 38.44 & 36.49 & 36.68 \\
\hline 11 & 23.29 & 24.88 & 21.08 & 20.87 \\
\hline 12 & 125.46 & 125.53 & 39.74 & 39.42 \\
\hline 13 & 139.17 & 139.26 & 42.28 & 42.58 \\
\hline 14 & 41.98 & 42.54 & 56.69 & 56.77 \\
\hline 15 & 29.14 & 28.63 & 24.29 & 24.09 \\
\hline 16 & 24.44 & 23.72 & 28.19 & 28.42 \\
\hline 17 & 47.54 & 48.02 & 56.01 & 56.24 \\
\hline 18 & 53.51 & 53.52 & 11.88 & 11.98 \\
\hline 19 & 39.01 & 39.39 & 19.38 & 19.18 \\
\hline 20 & 38.87 & 39.48 & 36.08 & 36.44 \\
\hline 21 & 30.48 & 31.08 & 18.79 & 18.79 \\
\hline 22 & 36.79 & 37.40 & 33.89 & 33.79 \\
\hline 23 & 28.37 & 29.33 & 26.08 & 25.91 \\
\hline 24 & 16.69 & 16.95 & 45.77 & 45.62 \\
\hline 25 & 15.79 & 17.48 & 29.11 & 29.76 \\
\hline 26 & 17.48 & 17.62 & 19.02 & 18.59 \\
\hline 27 & 23.44 & 23.91 & 19.77 & 19.55 \\
\hline 28 & 179.88 & 179.78 & 23.08 & 23.41 \\
\hline 29 & 17.09 & 17.45 & 12.05 & 12.14 \\
\hline 30 & 21.02 & 21.38 & & \\
\hline \multicolumn{5}{|l|}{ glu* } \\
\hline 1 & & & & 102.61 \\
\hline 2 & & & & 75.39 \\
\hline 3 & & & & 78.61 \\
\hline 4 & & & & 71.76 \\
\hline 5 & & & & 78.45 \\
\hline 6 & & & & 62.92 \\
\hline
\end{tabular}

${ }^{13} \mathrm{C}-\mathrm{NMR}$ measured in pyridine- $d_{5}(\mathbf{1}, \mathbf{2}$, and $\mathbf{4})$ and $\mathrm{CDCl}_{3}(\mathbf{3})$, respectively.

*glu: $\beta$-D-glucopyranose.

를 실시하여 4개의 분획(CFE-8-1-CFE-8-4)으로 나누었다. CFE$8-4(110 \mathrm{mg}, \mathrm{Ve} / \mathrm{Vt}=0.670-1.000)$ 을 ODS c.c. $(3 \times 7 \mathrm{~cm}$, acetone$\mathrm{H}_{2} \mathrm{O}=2: 1,2 \mathrm{~L}$ )를 실시하여 8개의 분획(CFE-8-4-1-CFE-8-4-8)으 로 나누었다고, 그 중 화합물 4 (CFE-8-4-7, $83 \mathrm{mg}, \mathrm{Ve} / \mathrm{Vt}=$ 0.476-0.882, TLC $\left.\left(\mathrm{SiO}_{2} \mathrm{~F}_{254}\right) \mathrm{R}_{\mathrm{f}} 0.42, \mathrm{CHCl}_{3}-\mathrm{MeOH}=10: 1\right)$ 을 분리하였다.

화합물 1 (ursolic acid): white powder (in $\mathrm{CHCl}_{3}$ ); m.p. $287^{\circ} \mathrm{C}$; $[\alpha]_{\mathrm{D}}^{25}+70.0^{\circ}\left(c \quad 0.7, \mathrm{CHCl}_{3}\right) ; \mathrm{EI} / \mathrm{MS} \mathrm{m} / z 456[\mathrm{M}]^{+}$; IR (KBr, v) $3360,1732,1648 \mathrm{~cm}^{-1}$; ${ }^{1} \mathrm{H}-\mathrm{NMR}$ spectrum $(400 \mathrm{MHz}$, pyridine$\left.d_{5}, \delta_{\mathrm{H}}\right) 5.48(1 \mathrm{H}$, br.s, $\mathrm{H}-12), 3.45(1 \mathrm{H}, \mathrm{dd}, J=10.0,5.4$ $\mathrm{Hz}, \mathrm{H}-3), 1.24$ (3H, s, H-23), 1.23 (3H, s, H-27), 1.04 
(3H, s, H-26), $1.02(3 \mathrm{H}, \mathrm{s}, \mathrm{H}-24), 1.01(3 \mathrm{H}, \mathrm{d}, J=6.4, \mathrm{H}-$ 30), $0.96(3 \mathrm{H}, \mathrm{d}, J=6.4, \mathrm{H}-29), 0.89$ (3H, s, H-25); ${ }^{13} \mathrm{C}-$ NMR (100 MHz, pyridine- $\left.d_{5}, \delta_{\mathrm{C}}\right)$; Table 1.

화합물 2 (corosolic acid): white powder (in $\mathrm{CHCl}_{3}$ ); m.p. $253^{\circ} \mathrm{C}$; $[\alpha]_{\mathrm{D}}^{25}+42.2^{\circ}\left(c \quad 0.1, \mathrm{CHCl}_{3}\right) ; \mathrm{EI} / \mathrm{MS} m / z 472[\mathrm{M}]^{+} ; \mathrm{IR}(\mathrm{KBr}, v)$ 3404, 1722, $1658 \mathrm{~cm}^{-1}$; ${ }^{1} \mathrm{H}-\mathrm{NMR}$ spectrum ( $400 \mathrm{MHz}$, pyridine$\left.d_{5}, \delta_{\mathrm{H}}\right) 5.43(1 \mathrm{H}$, br.s, $\mathrm{H}-12), 4.03(1 \mathrm{H}, \mathrm{ddd}, J=9.6$, 9.6, $3.2 \mathrm{~Hz}, \mathrm{H}-2), 3.34(1 \mathrm{H}, \mathrm{d}, J=9.6 \mathrm{~Hz}, \mathrm{H}-3), 1.23(3 \mathrm{H}, \mathrm{s}$, H-23), 1.19 (3H, s, H-27), 1.03 (3H, s, H-24), 1.01 (3H, s, H-25), 0.97 (3H, d, J=6.4, H-29), 0.96 (3H, s, H-26), 0.93 $(3 \mathrm{H}, \mathrm{d}, J=6.4, \mathrm{H}-30) ;{ }^{13} \mathrm{C}-\mathrm{NMR}\left(100 \mathrm{MHz}\right.$, pyridine- $\left.d_{5}, \delta_{\mathrm{C}}\right)$; Table 1 .

화합물 3 ( $\beta$-sitosterol): white powder (in $\mathrm{CHCl}_{3}$ ); m.p. $140^{\circ} \mathrm{C}$;

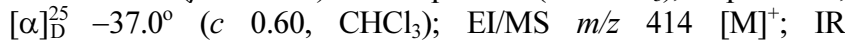
$(\mathrm{KBr}, v) 3413,1640 \mathrm{~cm}^{-1}$; ${ }^{1} \mathrm{H}-\mathrm{NMR}\left(400 \mathrm{MHz}, \mathrm{CDCl}_{3}, \delta_{\mathrm{H}}\right)$ $5.32(1 \mathrm{H}$, br.d, $J=3.6 \mathrm{~Hz}, \mathrm{H}-6), 3.51(1 \mathrm{H}, \mathrm{m}, \mathrm{H}-3), 1.02$ $(3 \mathrm{H}, \mathrm{s}, \mathrm{H}-19), 0.88(3 \mathrm{H}, \mathrm{d}, J=6.8 \mathrm{~Hz}, \mathrm{H}-21), 0.81(3 \mathrm{H}, \mathrm{d}$, $J=7.2 \mathrm{~Hz}, \mathrm{H}-29), 0.80(3 \mathrm{H}, \mathrm{d}, J=7.2 \mathrm{~Hz}, \mathrm{H}-26), 0.77(3 \mathrm{H}$, d, $J=6.8 \mathrm{~Hz}, \mathrm{H}-27), 0.64$ (3H, s, H-18); ${ }^{13} \mathrm{C}-\mathrm{NMR} \quad(100$ $\mathrm{MHz}$, pyridine- $\left.d_{5}, \delta_{\mathrm{C}}\right)$; Table 1 .

화합물 4 (daucosterol): white powder (in pyridine); m.p. $285^{\circ} \mathrm{C}$; $[\alpha]_{\mathrm{D}}^{25}-44.6^{\circ}$ (c 0.90 , pyridine); EI/MS $m / z \quad 577 \quad[\mathrm{M}]^{+} ;$IR $\left(\mathrm{KBr}\right.$, v) $3320,1645 \mathrm{~cm}^{-1}$; ${ }^{1} \mathrm{H}-\mathrm{NMR}\left(400 \mathrm{MHz}\right.$, pyridine- $d_{5}$, $\left.\delta_{\mathrm{H}}\right) 5.33(1 \mathrm{H}$, br.d, $J=3.6 \mathrm{~Hz}, \mathrm{H}-6), 5.05(1 \mathrm{H}, \mathrm{d}, J=7.6 \mathrm{~Hz}$, H-1), 3.95 (1H, m, H-3), 1.02 (3H, d, J=7.2 Hz, H-21), 0.94 (3H, s, H-19), $0.89(3 \mathrm{H}, \mathrm{d}, J=6.4 \mathrm{~Hz}, \mathrm{H}-26), 0.88(3 \mathrm{H}, \mathrm{d}$, $J=8.4 \mathrm{~Hz}, \mathrm{H}-27), 0.85$ (3H, t $J=6.8 \mathrm{~Hz}, \mathrm{H}-29), 0.64(3 \mathrm{H}$, $\mathrm{s}, \mathrm{H}-18) ;{ }^{13} \mathrm{C}-\mathrm{NMR}\left(100 \mathrm{MHz}\right.$, pyridine- $\left.d_{5}, \delta_{\mathrm{C}}\right)$; Table 1.

\section{결과 및 고찰}

이팝나무꽃을 $80 \% \mathrm{MeOH}$ 로 추출하여 얻어진 추출물에 대하여 용매의 극성에 따라 EtOAc, $n-\mathrm{BuOH}$ 및 $\mathrm{H}_{2} \mathrm{O}$ 로 순차 분획하고, 각 분획을 감압 농축하여 3 개의 분획을 얻었다. 3 개의 분획 중 EtOAc 분획으로부터 $\mathrm{SiO}_{2}$ 및 $\mathrm{ODS}$ c.c.를 반복 실시하여, 4종 의 화합물을 분리 및 동정하였다.

화합물 1 (white powder)는 EI/MS에서 $m / z 456[\mathrm{M}]^{+}$의 분자 이온 peak가 관측되어 분자량을 456 으로 결정하였다. IR 스펙 트럼으로부터 수산기 $\left(3360 \mathrm{~cm}^{-1}\right)$, carbonyl $\left(1732 \mathrm{~cm}^{-1}\right)$ 그리고 이중결합 $\left(1648 \mathrm{~cm}^{-1}\right)$ 의 작용기를 갖는 것으로 확인되었다. ${ }^{1} \mathrm{H}-$ NMR $\left(400 \mathrm{MHz}\right.$, pyridine- $\left.d_{5}\right)$ spectrum 에서 olefin methine proton signal $\left(\delta_{\mathrm{H}} 5.48,1 \mathrm{H}\right.$, br.s, $\left.\mathrm{H}-12\right)$ 과 oxygenated methine proton signal $\left(\delta_{\mathrm{H}} 3.45,1 \mathrm{H}, \mathrm{dd}, J=10.0,5.4 \mathrm{~Hz}, \mathrm{H}-3\right)$ 이 각각 1 개씩 관측되었다. 고자장영역에서 5개의 singlet methyl proton signal $\left(\delta_{\mathrm{H}} 1.24,3 \mathrm{H}, \mathrm{s}, \mathrm{H}-23 ; 1.23,3 \mathrm{H}, \mathrm{s}, \mathrm{H}-27 ; 1.04,3 \mathrm{H}, \mathrm{s}, \mathrm{H}-\right.$ $26 ; 1.02,3 \mathrm{H}, \mathrm{s}, \mathrm{H}-24 ; 0.89,3 \mathrm{H}, \mathrm{s}, \mathrm{H}-25)$ 이 관측되었고, 2 개 의 doublet methyl proton signal $\left(\delta_{\mathrm{H}} 1.01,3 \mathrm{H}, \mathrm{d}, J=6.4 \mathrm{~Hz}\right.$, $\mathrm{H}-30 ; 0.96,3 \mathrm{H}, \mathrm{d}, J=6.4 \mathrm{~Hz}, \mathrm{H}-29)$ 이 관측되었다. 또한 $\delta_{\mathrm{H}}$ 1.96-0.88에서 methine과 methylene proton signal이 다수 관측 되었다. 이를 종합하여 이중결합 1 개를 가진 ursane 골격의 triterpenoid로 예측하였다. ${ }^{13} \mathrm{C}-\mathrm{NMR}\left(100 \mathrm{MHz}\right.$, pyridine- $\left.d_{5}\right)$ spectrum에서 30 개의 탄소가 관측되어 triterpenoid 골격의 화합 물로 확인되었다. 저자장 영역에서는 carbonyl carbon signal

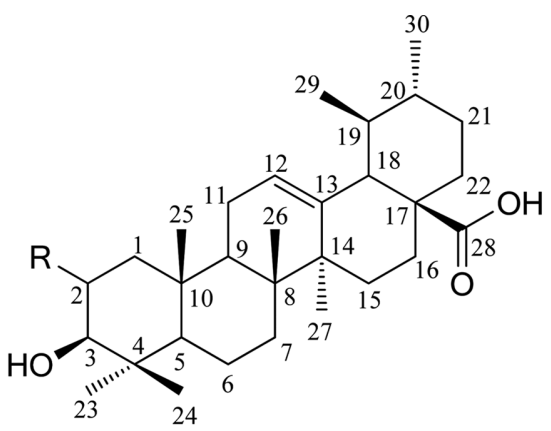

$\mathrm{R}$

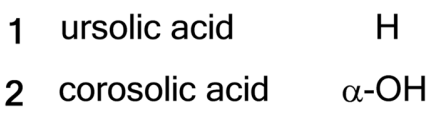<smiles>[R6]C1CC[C@@]2(C)C(=CCC3C2CC[C@]2(C)[C@@H]3CC[C@@H]2[C@H](C)CCC(CC)C(C)C)C1</smiles>

$\begin{array}{lll}3 & \beta \text {-sitosterol } & \mathrm{H} \\ 4 & \text { daucosterol } & \text { glu }\end{array}$

Fig. 1 Chemical structures of compounds (1-4) from the flowers of Chionanthus retusus Lindl. \& Paxton.

$\left(\delta_{\mathrm{C}} 179.88, \mathrm{C}-28\right)$, olefin quaternary carbon signal $\left(\delta_{\mathrm{C}} 139.17\right.$, $\mathrm{C}-13)$ 과 olefin methine carbon signal $\left(\delta_{\mathrm{C}} 125.46, \mathrm{C}-12\right)$ 이 각 각 한 개씩 관측되었으며, 수산기 영역에서도 한 개의 oxygenated methine carbon signal $\left(\delta_{\mathrm{C}} 78.15, \mathrm{C}-3\right)$ 이 관측되었 다. 또한 고자장 영역에서 quaternary carbon signal $\left(\delta_{\mathrm{C}} 47.54\right.$, C-17; 41.98, C-14; 41.64, C-8; 39.02, C-4; 36.97, C-10)과 methine carbon signal $\left(\delta_{\mathrm{C}} 55.84, \mathrm{C}-5\right.$; 53.51, C-18; 47.12, $\mathrm{C}-9 ; 39.01, \mathrm{C}-19 ; 38.87, \mathrm{C}-20$ )이 각각 5개씩 관측되었고, 이 밖에도, 9개의 methylene carbon signal $\left(\delta_{\mathrm{C}} 38.62, \mathrm{C}-1\right.$; 36.79 , C-22; 32.98, C-7; 30.48, C-21; 29.14, C-15; 28.24, $\mathrm{C}-2$; 24.44, C- 16 ; 23.29, C-11; 18.38, C-6)과 7개의 methyl carbon signal $\left(\delta_{\mathrm{C}} 28.37, \mathrm{C}-23 ; 23.44, \mathrm{C}-27 ; 21.02, \mathrm{C}-30\right.$; $17.48, \mathrm{C}-26 ; 17.09, \mathrm{C}-29 ; 16.69, \mathrm{C}-24 ; 15.79, \mathrm{C}-25)$ 이 관측 되었다. 이를 종합하여 문헌(Bang 등, 2008)과 비교한 결과, 화 합물 1은 ursolic acid으로 동정하였다(Fig. 1).

화합물 2 (white powder)는 $\mathrm{EI} / \mathrm{MS}$ 에서 $\mathrm{m} / z 472$ [M] ${ }^{+}$의 분 자이온 peak가 관측되어 분자량을 472 으로 결정하였고, 이는 화 합물 1보다 $m / z 16$ 이 큰 값으로, 화합물 1에 methylene 한 분 자가 oxygenated methine으로 치환된 구조임을 예상하였다. IR 스펙트럼으로부터 수산기 $\left(3404 \mathrm{~cm}^{-1}\right)$, carbonyl $\left(1722 \mathrm{~cm}^{-1}\right)$ 그 
리고 이중결합 $\left(1658 \mathrm{~cm}^{-1}\right)$ 의 작용기를 갖는 것으로 확인되었다. ${ }^{1} \mathrm{H}-\mathrm{NMR}$ 및 ${ }^{13} \mathrm{C}-\mathrm{NMR}$ 에서도 화합물 1과 비교하여 oxygenated methine 유래의 signal $\left(\delta_{\mathrm{H}} 4.03,1 \mathrm{H}\right.$, ddd, $J=9.6,9.6,3.2 \mathrm{~Hz}$, $\left.\mathrm{H}-2 ; \delta_{\mathrm{C}} 68.58, \mathrm{C}-2\right)$ 이 관측되었다. 따라서, 이 화합물을 ursolic acid의 2 번 위치의 methylene기가 oxygenated methine기로 치 환된 corosolic acid으로 구조동정하였고, 문헌(Lee 등, 2009)과 비교하여 확인하였다(Fig. 1).

화합물 3 (white powder)는 EI/MS에서 $m / z 414$ [M] 의 분 자이온 peak가 관측되어 분자량을 414 으로 결정하였다. IR 스 펙트럼으로부터 수산기 $\left(3413 \mathrm{~cm}^{-1}\right)$ 와 이중결합 $\left(1640 \mathrm{~cm}^{-1}\right)$ 의 작 용기를 갖는 것으로 확인되었다. ${ }^{1} \mathrm{H}-\mathrm{NMR}$ 및 ${ }^{13} \mathrm{C}-\mathrm{NMR}$ 를 문헌 (Sohn 등, 2009)과 비교 종합하여, 이 화합물을 sterol 화합물인 $\beta$-sitosterol으로 구조동정하였다(Fig. 1).

화합물 4 (white powder)는 $\mathrm{EI} / \mathrm{MS}$ 에서 $\mathrm{m} / z 577[\mathrm{M}]^{+}$의 분 자이온 peak가 관측되어 분자량을 577 으로 결정하였고, 이는 화 합물 3 보다 $m / z$ 163이 큰 값으로, 화합물 $\mathbf{3}$ 에 당한분자가 결합 된 구조임을 예상하였다. IR 스펙트럼으로부터 수산기 $\left(3320 \mathrm{~cm}^{-1}\right)$ 와 이중결합 $\left(1645 \mathrm{~cm}^{-1}\right)$ 의 작용기를 갖는 것으로 확인되었다. $\mathrm{NMR}$ 에서도 당에서 유래되는 signal들의 chemical shift $\left(\delta_{\mathrm{H}}\right.$ $5.05,1 \mathrm{H}, \mathrm{d}, J=7.6 \mathrm{~Hz}, \mathrm{H}-1 ; \delta_{\mathrm{C}} 102.61, \mathrm{C}-1 ; 78.61, \mathrm{C}-3$; 78.45, $\mathrm{C}-5 ; 75.39, \mathrm{C}-2 ; 71.76, \mathrm{C}-4,62.92, \mathrm{C}-6)$ 를 문헌과 비교하여, $\beta$-D-glucopyranose로 확인되었다. ${ }^{1} \mathrm{H}-\mathrm{NMR}$ 및 ${ }^{13} \mathrm{C}-\mathrm{NMR}$ 를 문헌 (Lyu 등, 2009)과 비교 종합하여, 이 화합물을 $\beta$-sitosterol의 C3 수산기에 $\beta$-D-glucopyranose가 결합하고 있는 화합물인 daucosterol으로 구조동정하였다(Fig. 1).

화합물 1-4은 이번에 이팝나무 꽃으로부터 처음으로 분리되 었다. Ursolic acid (1)는 암세포주에 대한 세포독성(Khiev 등, 2009)이 보고되었고, corosolic acid (2)는 ACAT 저해활성이 보 고 되었으며, $\beta$-sitosterol (3)과 daucosterol (4)은 암세포주에 대 한 세포독성(Lee 등, 2011)이 보고 되어 있다.

\section{초 록}

이팝나무(Chionanthus retusus Lindl. \& Paxton) 꽃은 실온에서 $80 \% \mathrm{MeOH}$ 수용액으로 추출하고 이 추출물을 $\mathrm{EtOAC}$ 분획, $n-\mathrm{BuOH}$ 분획, $\mathrm{H}_{2} \mathrm{O}$ 분획으로 나누었다. EtOAc 분획에 대하여 silica gel 및 ODS column chromatography를 반복 실시하여 4 종의 화합물을 분리 및 정제하였다. nuclear magnetic resonance, infrared, 및 Electronic ionization mass spectrometer 등을 해석 하여, ursolic acid (1), corosolic acid (2), $\beta$-sitosterol (3), 그 리고 daucosterol (4)로 구조동정 하였다. 분리한 4종의 화합물 은 이팝나무 꽃으로부터 이번 실험에서 처음 분리 되었다.

Keywords 이팝나무 · 꽃 · nuclear magnetic resonance · sterol - triterpenoid

감사의 글 본 연구는 중소기업청에서 지원하는 기능성 화장품 지표성분 분 석법 확립 및 소재 표준화 연구(과제번호: S2091482)로 수행된 과제로 연구 비 지원에 감사드립니다.

\section{References}

Bang MH, Cho JG, Song MC, Lee DY, Han MW, Chung HG et al. (2008) Development of biologically active compounds from edible plant sources XXII. Triterpenoids from the aerial parts of Sajabalssuk (Artemisia princeps PAMPANINI). J Korean Soc Appl Biol Chem 51, 223-7.

Khiev P, Cai XF, Chin YW, Ahn KS, Lee HK, and Oh SR (2009) Cytotoxic terpenoids from the methanolic extract of Bridelia cambodiana. $J$ Korean Soc Appl Biol Chem 52, 626-31.

Kim TJ (1996) In Korean resources plants (III), Seoul National University Press, Korea.

Kwak JH, Kang MW, Roh JH, Choi SU, and Zee OP (2009) Cytotoxic phenolic compounds from Chionanthus retusus. Arch Pharm Res 32, $1681-7$.

Lee DG, Park JH, Yoo KH, Chung IS, Lee YH, Lee JK et al. (2011) 24Ethylcholesta-4,24(28)-dien-3,6-dione from Osmanthus fragrans var. aurantiacus flowers inhibits the growth of human colon cancer cell line, HCT-116. J Korean Soc Appl Biol Chem 54, 206-10.

Lee DY, Jung L, Lyu HN, Jeong TS, Lee YH, and Baek NI (2009) Triterpenoids from the fruits of Cornus kousa Burg. as human Acyl-Coa: Cholesterol acyltransferase inhibitors. Food Sci Biotechnol 18, 223-7.

Lee JS, Lee KH, and Oh CJ (1996) In New Woody Plant Flora of Korea, Haksulcenter, Korea.

Lyu HN, Park JH, Kwak HY, Lee DY, Cho JG, Kim SY et al. (2009) Isolation of steroids from the Kalopanacis cortex. J Appl Biol Chem 52, 41-4.

Sohn BH, Park JH, Lee DY, Cho JG, Kim YS, Jung IS et al. (2009) Isolation and identification of lipids from the silkworm (Bombyx mori) droppings. J Korean Soc Appl Biol Chem 52, 336-41. 